\title{
Grippeimpfung bei Typ-2-Diabetes schützt vor Klinik und Schlimmerem
}

Hintergrund und Fragestellung: Die saisonale Grippe kann die Gesundheit stark gefährden. Die Autoren wollten die Effektivität einer Grippeimpfung zur Vermeidung von Krankenhauseinweisungen aufgrund von akuten kardiovaskulären und respiratorischen Symptomen sowie die Gesamtmortalität bei Patienten mit Typ-2-Diabetes untersuchen.

Patienten und Methoden: Die Autoren führten eine retrospektive Kohortenstudie durch, für die primäre und sekundäre Daten aus einer englischen Datenbank (mit dem Namen Clinical Practice Research Datalink) verwendet wurden, die eine 7-Jahres-Spanne zwischen 2003/4 und 2009/10 erfasste. Es wurden 124.503 Erwachsene mit Typ-2-Diabetes betrachtet. Endpunkte umfassten die Aufnahme ins Krankenhaus wegen eines akuten Myokardinfarkts

\section{Originalie}

Vamos EP, Pape UJ, Curcin V et al. Effectiveness of the influenza vaccine in preventing admission to hospital and death in people with type 2 diabetes. CMAJ. 2016 Oct 4;188(14):E34-51. (MI), Schlaganfall, Herzinsuffizienz oder Pneumonie/ Influenza sowie Tod. Mittels Poisson-Regression wurde die Inzidenzdaten-Ratios (IRR) für Influenza- und Nicht-Influenza-Zeiten ermittelt und für Kohorten ge- schätzt, die die Impfung erhalten hatten oder nicht. Schätzungen zur Sommerzeit, die eine niedrige Influenza Aktivität aufweist, wurden für die Adjustierung von restlichen Confoundern verwendet.

Ergebnisse: Während der 7-Jahres-Periode wurden 623591 Personenjahre beobachtet. Personen, die Impfungen erhalten hatten, waren älter und hatten häufiger Begleiterkrankungen im Vergleich zu nicht Geimpften. Nach der Adjustierung für Kovariaten und residuelles Confounding war die Grippeimpfung mit einer signifikant niedrigeren Krankenhausaufnahmerate aufgrund von Schlaganfall (IRR 0,70, 95\%-Konfidenzintervall [KI] 0,53-0,91), Herzinsuffizienz (IRR 0,78, 95\%-KI 0,65-0,92) und Pneumonie oder Influenza (IRR 0,85, 95\%-KI 0,74-0,99), und auch Gesamtmortalität (IRR 0,76, 95\%-KI 0,65-0,83) während der Grippesaison assoziiert. Der akute Myokardinfarkt war nicht signifikant (IRR 0,81, 95\%-KI 0,62-1,04) assoziiert.

Schlussfolgerungen: Bei dieser Kohorte mit Typ-2-DiabetesPatienten war die Grippeimpfung mit einer reduzierten Rate an Krankenhausaufnahmen wegen spezifischer kardiovaskulärer Ereignisse assoziiert. Bemühungen sollten angestrengt werden, um die Impfrate in dieser wichtigen Zielgruppe im Rahmen einer sekundären Prävention zu verbessern.

\section{- Kommentar von Prof. Dr. med. Nanette C. Schloot}

\section{Evidenz und Plädoyer für Grippeimpfung bei Diabetespatienten}

Im Winter sterben noch immer viele Menschen an den Folgen einer Influenzaerkrankung. In Europa konzentrieren sich die Impfungen gegen Grippeviren vor allem auf ältere Personen und solche, die ein hohes Risiko der Ansteckung und Übertragung haben wie Bedienstete im Gesundheitssystems. Bei Patienten mit Diabetes, deren Risiko für influenzaassoziierte Komplikationen besonders hoch ist, wurde wiederholt vermutet, dass sie vermindert auf die Influenza-Vakzine ansprechen könnten. Allerdings gibt es kaum Studien, in denen die Impferfolge bei Typ-2-Diabetes systematisch in prospektiven Studien untersucht wurden. Außerdem ist zu bezweifeln, dass solch eine Studie ethisch angebracht wäre. Die Autoren der aktuellen britischen Studie adressierten nun diese Frage anhand epidemiologischer Daten mit einer großen Zahl von Patienten und fanden, dass in der Tat, Typ-2-Diabetes-Patienten von der Grippeimpfung profitieren und danach weniger häufig ins Krankenhaus müssen oder sterben. Hervorzuheben ist die gründliche Adjustierung auf Parameter wie Begleiterkrankungen, Diabetesdauer, Raucherstatus etc.. Dieses Resultat sollte in der Diabetologie zu erhöhten Anstrengungen führen, Patienten gegen Grippe zu impfen und darüber aufzuklären. Auch wenn eine entsprechende prospektive, randomisierte Studie hierzu noch aussteht.

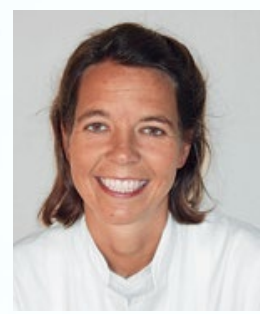

Prof. Dr. med. Nanette C. Schloot

Deutsches Diabetes-Zentrum, Institut für Klinische Diabetologie an der Heinrich-Heine-Universität Düsseldorf Auf'm Hennekamp 65, 40225 Düsseldorf Nanette.Schloot@DDZ.uni-duesseldorf.de 